\title{
Somatic Mutations in Exons 10,11, and 16 of the RET Protooncogene in Medullary Thyroid Carcinoma Patients
}

\author{
Elham Shakiba $^{\circledR}{ }^{\circledR}$, Mehdi Hedayati $^{2}$, Ahmad Majd ${ }^{1}$, Monireh Movahedii ${ }^{*}$ \\ ${ }^{1}$ Department of Cellular and Molecular Biology, Faculty of Biological Sciences, North Tehran Branch, Islamic Azad \\ University, Tehran, Iran \\ ${ }^{2}$ Cellular and Molecular Endocrine Research Center, Research Institute for Endocrine Sciences, Shahid Beheshti University \\ of Medical Sciences, Tehran, Iran
}

\author{
*Correspondence to \\ Monireh Movahedi, \\ Tel: +989127391290 \\ Email: mon-movahedi@yahoo.com
}

Received May 31, 2020

Accepted July 18, 2020

Published online 30 Septemeber 2020

\begin{abstract}
Introduction: Medullary thyroid carcinoma (MTC) comprises nearly 5\% of all cases of thyroid cancer (TC). Aberrant activation of RET (rearranged during transfection) signaling via somatic mutations is the basic molecular mechanism of MTC tumorigenicity. In this study, we determined the incidence of RET gene mutations in exons 10,11 , and 16 in Iranian patients.

Methods: A total of 33 patients undergoing thyroidectomy at Imam Khomeini hospital of Tehran, Iran and diagnosed with MTC were enrolled. For investigating mutations in exons 10, 11, and 16, DNA was extracted from tumor tissues, and the genes were amplified by polymerase chain reaction (PCR) and then sequenced.

Results: Out of 33 patients, 20 (60.6\%) subjects had mutations in one of the examined exons (10, 11, and 16). According to our results, the "ATG918ACG" mutation in codon 918 had the highest rate.

Conclusion: Testing RET mutations can be beneficial in clinical evaluation and treatment management of MTC patients.

Keywords: Thyroid, Caner, Medullary thyroid carcinoma, RET gene, Somatic mutations
\end{abstract}

Please cite this article as follows: Shakiba E, Hedayati M, Majd A, Movahedi M. Somatic mutations in exons 10,11 , and 16 of the ret protooncogene in medullary thyroid carcinoma patients. Int J Basic Sci Med. 2020;5(3):108113. doi: $10.34172 /$ ijbms.2020.19.

\section{Introduction}

Although palpable thyroid nodules (PTNs) may be found in around $4-7 \%$ of general population, around $5 \%$ of these PTNs transform to cancerous masses. ${ }^{1}$ Thyroid cancer (TC) is a common tumor of endocrine system comprising 5\%-10\% of all thyroid nodules. Epidemiological studies around the world show a soaring pattern in TC diagnosis. Among all types of human tumors, the incidence of TC has particularly increased in recent decades. ${ }^{1}$ Around $7.5 \%-10 \%$ of TCs originate from parafollicular cells, which is known as medullary thyroid carcinoma (MTC). ${ }^{1}$ According to the Iranian Cancer Institute, TC accounts for $1.8 \%$ of all cancers among Iranian people. Moreover, TC is the seventh, fourteenth, and eleventh most common cancer in Iranian women, men, and general population, respectively. ${ }^{2}$

Regarding histopathology, differentiated and non-differentiated thyroid tumors are two main categories. The later includes two main subcategories: anaplastic thyroid cancer (ATC) and MTC. Thyroid tumors usually originate from follicular cells, while MTCs develop from parafollicular cells. ${ }^{3}$

MTCs which originate from $\mathrm{C}$ or parafollicular cells that produce thyroid calcitonin comprise $5-7 \%$ of all thyroid tumors ${ }^{4,5}$; while majority of them are sporadic, about one-fourth are hereditary. ${ }^{5}$ Hereditary types include multiple endocrine neoplasia (MEN-2A and MEN-2B) and familial MTC (FMTC). Familial types are inherited as autosomal dominant traits and are characterized with RET mutations. ${ }^{6-8}$ Clinically, FMTC is characterized by a complete penetrance but different expressions. MTC presents in all, and around half develop MEN-2B or MEN-2A pheochromocytoma. A ratio of MEN-2A patients suffer from parathyroid hyperplasia. ${ }^{9}$

Receptor tyrosine kinases (RTKs) are a

(c) 2020 The Author(s); Published by Zabol University of Medical Sciences. This is an open-access article distributed under the terms of the Creative Commons Attribution License (http://creativecommons.org/licenses/by/4.0), which permits unrestricted use, distribution, and reproduction in any medium, provided the original work is properly cited. 
large family of receptors activated by binding to ligands. RTKs mediate cell proliferation, differentiation, survival, and motility. RET (rearranged during transfection) at 10q11.2 was amongst the first RTKs, which was identified to be associated with neoplasia. RET has been considered to be responsible for all types of inherited TCs. Point mutations in sporadic MTC lead to gain-of-function mutation and tumorigenesis. ${ }^{10}$ The molecular basis of MTC tumors is the inappropriate activation of RET signaling due to a mutation in RET, which does not exist in tumors derived from thyroid follicular cells. ${ }^{11}$

Mutations in six exons of RET $(10,11,13,14,15,16)$, which correspond to cysteine-rich and tyrosine kinase domains of the receptor, comprise more than 95\% of cases, and all first-degree relatives of newly diagnosed MTC patients should be screened for these mutations. ${ }^{12}$ Somatic mutations in codon 918 are the most common types and are associated with aggressive MTC. ${ }^{12}$

The RET RTKs are commonly mutated in thyroid tumors and MTC. ${ }^{13}$ RET-activating mutations confer oncogenic activity and play a key role in apoptosis, viability, and proliferation of cancer cells thorough pathways such as PI3K/AKT. Therefore, RET serves as a potential target for treating MTC patients. ${ }^{13}$

Various RET gain-of-function mutations, especially in exon 10 (codons 609, 611, 618 and 620), exon 11 (codons 630 and 634), and exon 16 (codon 918) are well-known to be linked with FMTC, MEN-2B, and MEN-2A. ${ }^{14}$, 15 Accordingly, in this study, we investigated somatic mutations in these exons in Iranian MTC patients.

\section{Materials and Methods}

In this case-control study, 33 patients with MTC (20 males and 13 females) were investigated. The margins of tumors from the same patients were differentiated from tumors by a pathologist and considered as controls. The case and control groups were exactly matched for age, sex, and clinical stages.

The samples were collected from paraffin-embedded tissue blocks at Imam Khomeini Hospital of Tehran, Iran, during 2008-2012. The patients' specifications were extracted from the reports of archived files. Patients were selected based on the histological subtypes of tumors. Each paraffin-embedded tissue block was cut into 5-micron sections by a microtome machine, and the tissue sections were placed into $1.5 \mathrm{~mL}$ microtubes.

\section{DNA Extraction}

DNA was extracted from the samples using the QIAamp DNA FFPE Tissue DNA extraction kit (Qiagen, Germany) according to the kit protocol. Before DNA extraction, the tissues were washed with xylene and then absolute alcohol (Sigma, St. Louis, MO, USA).

The quality of the extracted DNA was assessed with NanoDrop ${ }^{\circledast}$ ND-1000 (Thermo Scientific, USA). The samples were then reached to $100 \mathrm{ng} / \mu \mathrm{L}$ concertation by adding distilled water. The quality of DNA was also checked by running $5 \mu \mathrm{L}$ of the extracted DNA on $1 \%$ agarose gel.

\section{PCR for Amplifying Exons 10, 11, and 16}

In order to amplify the exons 10, 11, and 16 of RET protooncogene, the primers (F5'GCGCCCCAAGGAGGCTGATGC3') and (R5'CGTGGTGGTCCCGCCCGCC3') for exon 10, the primers (F5'CCTCTGCGGTGCCAAGCCTC3') and (R5'CACCGGAAGAGAGAGTAGCTG3') for exon 11, and the primers (F 5'GTGCCCAGGAGTGTCTACCA3') and (R5'CAGGACCACAGGAGGGTAAC3 ') for exon 16 were applied. Polymerase chain reaction (PCR)was performed in a $30 \mu \mathrm{L}$ volume containing $1 \mu \mathrm{L}$ of each forward and reverse primer $(10 \mu \mathrm{M}), 1 \mu \mathrm{l}$ DNA (100 $n g / \mu L)$, Taq PCR PreMix (Bioneer, South Korea) and $27 \mu \mathrm{L}$ water. Reactions were performed in an automatic thermocycler (peqSTAR 96X HP, Peqlab Co., Germany).

PCR reaction was conducted considering an initial denaturation at $94^{\circ} \mathrm{C}$ for 10 minutes, and 30 cycles at $94^{\circ} \mathrm{C}$ for 45 seconds (denaturation), $66^{\circ} \mathrm{C}$ for 45 seconds (annealing), and $72^{\circ} \mathrm{C}$ for 30 seconds (extension). A final extension phase at $72^{\circ} \mathrm{C}$ was also held for 10 minutes.

After PCR, the amplicons were visualized on $8 \%$ polyacrylamide gel and stained with silver nitrate. After confirming the amplification of exons, they were sequenced and evaluated using Chromas software (version 2.6.4). The resultant peaks were blasted against reference sequences from the gene bank (http://www.ncbi.nlm.nih. gov/blast/Blast.cgi) using the Chromas software (version 2.6.4).

\section{Results}

The samples of 33 MTC patients (20 males and 13 females) were analyzed, of which $20(60.6 \%)$ cases showed mutations in at least one exon of RET gene (Figure 1). Table 1 shows the patients' clinical and demographic features.

\section{RET Protooncogene Mutations}

Twenty patients $(60.6 \%)$ were identified with mutations in exons 10,11 , and 16 (Table 2), and 13 patients did not have any mutations in these exons.

Out of 20 individuals (10 females and 10 males) with mutations in exons 10,11 , and 16 , only one 25 -year-old male had mutations in both exons 16 and 10 (Figure 2).

The "ATG918ACG" mutation in the codon 918 of exon

Table 1. The Patients' Features

\begin{tabular}{lcc}
\hline Cancer Type & $\begin{array}{c}\text { Age }(\mathbf{y})(\text { mean } \\
\pm \text { SD) }\end{array}$ & $\begin{array}{c}\text { Male: Female } \\
\text { Ratio }\end{array}$ \\
\hline MTC & $41.6 \pm 17.3$ & $20: 13$ \\
MTC without RET mutation $(\mathrm{n}=13)$ & $43.6 \pm 19.7$ & $8: 5$ \\
MTC with RET mutation $(\mathrm{n}=20)$ & $41.8 \pm 15.2$ & $10: 10$ \\
\hline
\end{tabular}


Table 2. Mutations Observed in Exons 10, 11, and 16 of RET Protooncogene

\begin{tabular}{lcc}
\hline Mutations & Wild Sequence & Mutated Sequence \\
\hline C634F & TTC & TGC \\
C611S & AGC & TGC \\
C618R & CGC & TGC \\
C609S & AGC & TGC \\
M918T & ACG & ATG \\
\hline
\end{tabular}

C:Cys, S:ser, R: Arg , F: phe, M: met, T: Thr.

16 was the most common mutation (13 out of 20). Seven mutations were identified in exon 10, and "TGC618CGC" was the most common one. The "TGC634TTC" mutation in exon 11 was observed in two individuals.

Chromatograms obtained from sequencing of amplicons with mutations in exons 10, 11, and 16 have been shown in Figures 3, 4, and 5, respectively.

\section{Discussion}

Somatic RET mutations are detected in about $30 \$-50 \%$ of sporadic and $92 \%$ of inherited MTC cases. ${ }^{16,17}$ This indicates the significant role of RET in MTC pathogenesis. All three clinically distinct types of MTC (i.e. MEN-2A, MEN-2B, and FMTC) are characterized with aggressive histopathology and high death rate in malignancies of thyroid. ${ }^{18}$ In this regard, investigating RET somatic mutations in MTC tumors can help predict prognosis and response to $R E T$-inhibitors. ${ }^{19}$

These mutations elevate the rate of metastasis to lymph node and distant locations, and decreased survival in patients. ${ }^{20}$ In the present study, from 33 patients with MTC (21 to 70 years), 20 cases (10 males and 10 females; age range: $24-65$ years) showed mutations in exons 10,11 , and 16 of RET gene, and 13 patients showed no mutations. In another study, it was reported that the percentage of patients with RET mutations in tumor tissues was $50 \% .^{21}$ Schulten et al also studied 13 patients with MTC, of whom 5 showed mutations in exon 16, and two patients had mutations in exon 10 (C620S and C618S). Therefore, it is important to screen MTC patients in terms of RET gainof-function mutations. Sporadic MTCs are particularly associated with M918T mutation in exon 16, which is associated with the highest risk of tumor invasion. Schulten et al reported that RET mutations in exons 10 and 16 were detected in seven out of 13 MTC patients. Exon 10 codes the extracellular cysteine-rich domain of RET, and its mutations result in ligand-independent dimerization and activation of the receptor. Hereditary mutations in exon 10 (mostly C620S and C618S) are associated with MEN-2A. In contrast, exon 16 encodes the intracellular tyrosine kinase domain. The M918T is the most common inherited mutation of exon 16, which is associated with MEN-2B. ${ }^{22}$ In our study, mutations in exon 16 were the most common alternations of RET observed in $39.4 \%$ of patients, and exon 10 mutations were also identified in
$21.20 \%$ of patients. Out of patients with mutations, one 25-year-old male had mutations in both exons 16 and 10. The "ATG918ACG" in the codon 918 of exon 16 was the most common mutation observed in this exon. Seven mutations (including TGC609AGC, TGC618CGC, and TGC611AGC) were observed in exon 10, among them codon 618 mutation showed the highest rate. Finally, TGC634TTC mutation in exon 11 was observed in two

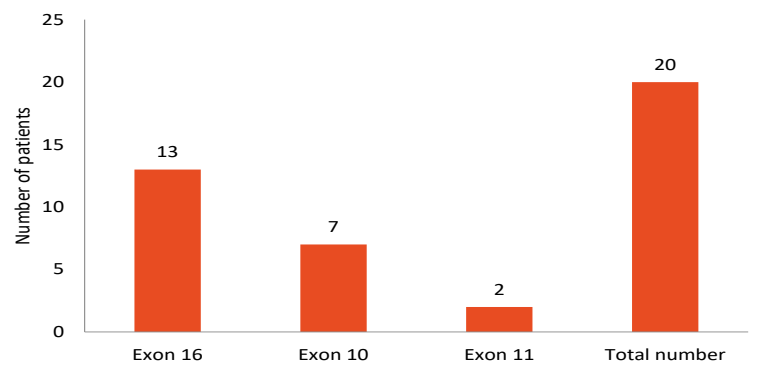

Figure 1. RET Protooncogene Mutations in Patients With Medullary Thyroid Cancer.

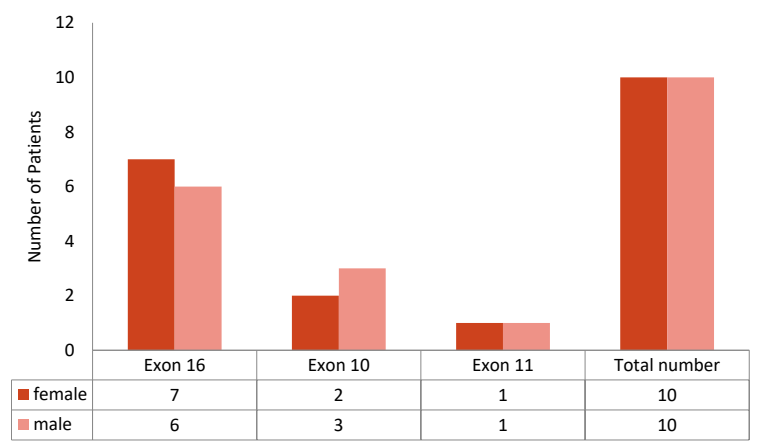

Figure 2. Number of Females and Males With MTC Who Showed Mutations in Exons 10, 11, and 16 of RET Protooncogene.

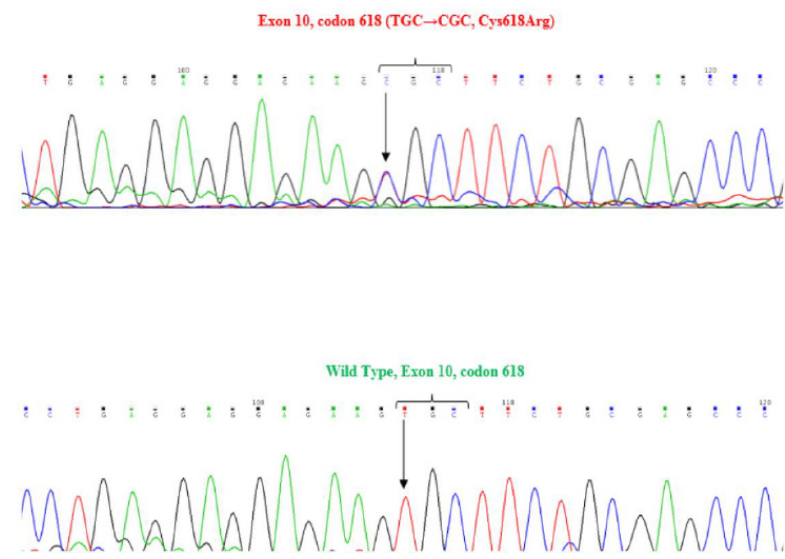

Figure 3. The 618 Codon Mutation in Exon 10 of RET Gene Characterized with the Substitution of Cysteine With Arginine (up) in Comparison with the Normal Sequence (down). 

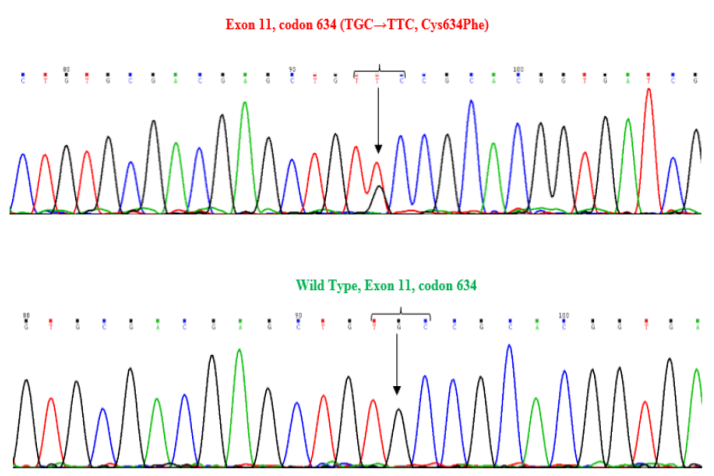

Figure 4. The Mutation in the 634 Codon of Exon 11 (up) in Comparison With Reference Sequence (down).

The mutation is characterized with the substitution of cysteine with phenylalanine.
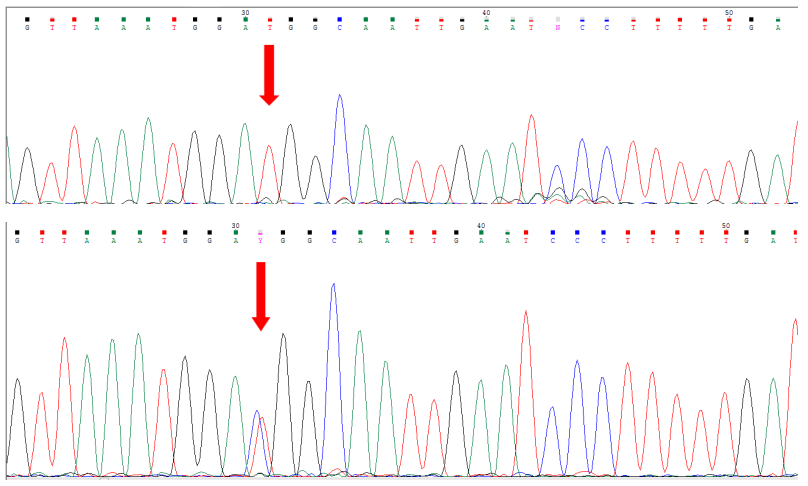

Figure 5. Sequencing of Exon 16 Shows the Mutation Converting ATG to ACG in the 918 Codon of Exon 16 (up) in Comparison With Normal Sequence (down).

individuals. Missense mutations in each of 6 codons of RET gene that encode cysteine amino acids (codons 609, 611,618 , and 620 of exon 10 and codons 630 and 634 of exon 11) forming the extracellular cysteine-rich domain of the RTK lead to constitutive homodimerization and autophosphorylation of specific tyrosine residues in RET intracellular kinase domain. ${ }^{23}$ In patients with MEN$2 \mathrm{~B}$, mutations are generally limited to RET intracellular tyrosine kinase domain (mainly includes codon 918 of exon 16$){ }^{24}$

RET germ line mutations lead to hereditary subtypes while somatic mutations lead to sporadic MTC. ${ }^{15}$ More than $90 \%$ of FMTC and MEN-2A patients show missense mutations in either 609, 611, 618, 620 (in exon 10) or 630 and 634 (in exon 11) codons corresponding to the protected extracellular cysteine-rich domains of RET receptor. ${ }^{25,26}$ In MEN-2B subtype, mutations are most commonly observed in the codon 918 of exon $16 .{ }^{27}$ As mentioned, mutations in extracellular cysteine-rich domains (i.e. $609,611,618$, and 620 codons of exon 10, and 630, and 634 codons of exon 11) lead to RET tyrosine kinase autophosphorylation in the absence of ligands. In addition, mutations in the intracellular tyrosine kinase region (such as exon 16; codon 918) do not affect the receptor dimerization, but activate intracellular signaling pathways which lead to tumorigenesis. ${ }^{28}$ Furthermore, insertions and deletions have been detected in exons 10 , 11,13 , and 15 , though they are less common. ${ }^{29}$

In general, investigating RET mutations is necessary for clinical assessment of sporadic MTC and MEN-2 carriers. In addition, based on the nature of RET mutations, it is possible to estimate invasive behavior of tumors. Recent studies show that the effect of different MTC inhibitors is related to the type of RET mutations, highlighting their importance in clinical setting. ${ }^{22}$ Screening RET gene mutations in sporadic MTC cases has resulted in variable frequencies from $12 \%$ to $100 \%$. The Met918Thr mutation is observed in 5\%-66\% of MTC patients. ${ }^{30}$ The number of screened exons and variable sample sizes can explain the variable rates of RET mutations in sporadic MTC. Sampling methods could also have been effective in causing these differences. The Met918Thr somatic mutation in the tyrosine kinase domain of exon 16 of RET may exert an adverse clinical outcome in sporadic MTC. In a study by Moura et al, 51 patients with MTC were evaluated of whom $33(64.7 \%)$ revealed somatic mutations in RET [3 (9.1\%) patients in exon 10, 7 (21.2\%) patients in exon 11, and $20(60.6 \%)$ patients in exon $16]$. With respect to RET mutations, these researchers identified three groups of patients: (1) those with mutations in exon 16,2 ) those with mutations in other exons, and (3) patients without mutations. Patients in the first group demonstrated a higher prevalence of lymph node metastasis and multifocal tumors. ${ }^{30}$

\section{Conclusion}

RET somatic mutations result in more aggressiveness and poorer prognosis in patients with MTC. Thus, detecting these mutations can be helpful in the diagnosis, prognosis, and follow-up of these patients. In the present study, out of $33(60.6 \%)$ patients, 20 cases were identified with mutations in exons 10,11 , and 16 . This shows the necessity of screening RET mutations in MTC patients.

\section{Ethical Approval}

This article has been extracted from a $\mathrm{PhD}$ thesis in the field of biochemistry approved by the Islamic Azad University, North Tehran Branch, Iran. The study was approved by the Ethics Committee of the Shahid Beheshti University (IR.SBMEU.REC.1395.10462).

\section{Conflict of Interest Disclosure}

The authors declare no conflict of interests.

\section{Authors' Contributions}

$\mathrm{MH}$ : Supervision, concept, and design; ESH: Clinical studies and data gathering; MM and AM: Drafting and critically revising the manuscript; ESH: Data collection and analysis. 


\section{Acknowledgments}

The authors would like to thank reviewers for their cooperation by reviewing this article and advising valuable comments.

\section{References}

1. Romei C, Elisei R. RET/PTC translocations and clinicopathological features in human papillary thyroid carcinoma. Front Endocrinol (Lausanne). 2012;3:54. doi:10.3389/fendo.2012.00054

2. Taghavi Kojidi H, Farzadfar F, Peykari N, et al. A comprehensive study on national and sub national trend in thyroid cancer prevalence in the Iranian population, 1990-2010. Iranian Journal of Diabetes and Metabolism. 2016;15(2):91-100. [Persian].

3. Rodríguez-Rodero S, Fernández AF, Fernández-Morera JL, et al. DNA methylation signatures identify biologically distinct thyroid cancer subtypes. J Clin Endocrinol Metab. 2013;98(7):2811-2821. doi:10.1210/jc.2012-3566

4. Pacini F, Castagna MG, Brilli L, Pentheroudakis G. Thyroid cancer: ESMO Clinical Practice Guidelines for diagnosis, treatment and follow-up. Ann Oncol. 2012;23 Suppl 7:vii110-119. doi:10.1093/annonc/mds230

5. Leboeuf R, Langlois MF, Martin M, Ahnadi CE, Fink GD. "Hook effect" in calcitonin immunoradiometric assay in patients with metastatic medullary thyroid carcinoma: case report and review of the literature. J Clin Endocrinol Metab. 2006;91(2):361-364. doi:10.1210/jc.2005-1429

6. Hofstra RM, Landsvater RM, Ceccherini I, et al. A mutation in the RET proto-oncogene associated with multiple endocrine neoplasia type $2 \mathrm{~B}$ and sporadic medullary thyroid carcinoma. Nature. 1994;367(6461):375-376. doi: $10.1038 / 367375 \mathrm{a} 0$

7. Mulligan LM, Kwok JB, Healey CS, et al. Germ-line mutations of the RET proto-oncogene in multiple endocrine neoplasia type 2A. Nature. 1993;363(6428):458460. doi:10.1038/363458a0

8. Mulligan LM, Eng C, Healey CS, et al. Specific mutations of the RET proto-oncogene are related to disease phenotype in MEN 2A and FMTC. Nat Genet. 1994;6(1):70-74. doi:10.1038/ng0194-70

9. Wells SA Jr, Santoro M. Targeting the RET pathway in thyroid cancer. Clin Cancer Res. 2009;15(23):7119-7123. doi:10.1158/1078-0432.ccr-08-2742

10. Phay JE, Shah MH. Targeting RET receptor tyrosine kinase activation in cancer. Clin Cancer Res. 2010;16(24):59365941. doi:10.1158/1078-0432.ccr-09-0786

11. Hofstra RM, Landsvater RM, Ceccherini I, et al. A mutation in the RET proto-oncogene associated with multiple endocrine neoplasia type $2 \mathrm{~B}$ and sporadic medullary thyroid carcinoma. Nature. 1994;367(6461):375-376. doi: $10.1038 / 367375 \mathrm{a} 0$

12. Kouvaraki MA, Shapiro SE, Perrier ND, et al. RET protooncogene: a review and update of genotype-phenotype correlations in hereditary medullary thyroid cancer and associated endocrine tumors. Thyroid. 2005;15(6):531544. doi:10.1089/thy.2005.15.531

13. Santarpia L, Ye L, Gagel RF. Beyond RET: potential therapeutic approaches for advanced and metastatic medullary thyroid carcinoma. J Intern Med. 2009;266(1):99-
113. doi:10.1111/j.1365-2796.2009.02112.x

14. Boikos SA, Stratakis CA. Molecular mechanisms of medullary thyroid carcinoma: current approaches in diagnosis and treatment. Histol Histopathol. 2008;23(1):109-116. doi:10.14670/hh-23.109

15. Elisei R, Cosci B, Romei C, et al. Prognostic significance of somatic RET oncogene mutations in sporadic medullary thyroid cancer: a 10-year follow-up study. J Clin Endocrinol Metab. 2008;93(3):682-687. doi:10.1210/jc.2007-1714

16. Agrawal N, Jiao Y, Sausen M, et al. Exomic sequencing of medullary thyroid cancer reveals dominant and mutually exclusive oncogenic mutations in RET and RAS. J Clin Endocrinol Metab. 2013;98(2):E364-369. doi:10.1210/ jc.2012-2703

17. Eng C, Clayton D, Schuffenecker I, et al. The relationship between specific RET proto-oncogene mutations and disease phenotype in multiple endocrine neoplasia type 2 . International RET mutation consortium analysis. JAMA. 1996;276(19):1575-1579.

18. Lanzi C, Cassinelli G, Nicolini V, Zunino F. Targeting RET for thyroid cancer therapy. Biochem Pharmacol. 2009;77(3):297-309. doi:10.1016/j.bcp.2008.10.033

19. Elisei R, Molinaro E, Agate L, et al. RET oncogene and thyroid carcinoma. J Genet Syndr Gene Ther. 2014;5(1):214. doi:10.4172/2157-7412.1000214

20. Quayle FJ, Moley JF. Medullary thyroid carcinoma: management of lymph node metastases. Curr Treat Options Oncol. 2005;6(4):347-354. doi:10.1007/s11864-005-0038-6

21. Ball DW. Medullary thyroid cancer: therapeutic targets and molecular markers. Curr Opin Oncol. 2007;19(1):18-23. doi:10.1097/CCO.0b013e32801173ea

22. Schulten HJ, Al-Maghrabi J, Al-Ghamdi K, et al. Mutational screening of RET, HRAS, KRAS, NRAS, BRAF, AKT1, and CTNNB1 in medullary thyroid carcinoma. Anticancer Res. 2011;31(12):4179-4183.

23. Arlt DH, Baur B, Wagner B, Höppner W. A novel type of mutation in the cysteine rich domain of the RET receptor causes ligand independent activation. Oncogene. 2000;19(30):3445-3448. doi:10.1038/sj.onc.1203688

24. Iwashita T, Asai N, Murakami H, Matsuyama M, Takahashi M. Identification of tyrosine residues that are essential for transforming activity of the ret proto-oncogene with MEN2A or MEN2B mutation. Oncogene. 1996;12(3):481487.

25. Chang JM, Hwang SJ, Kuo HT, et al. Fatal outcome after ingestion of star fruit (Averrhoa carambola) in uremic patients. Am J Kidney Dis. 2000;35(2):189-193. doi:10.1016/s0272-6386(00)70325-8

26. Marsh DJ, Andrew SD, Eng C, et al. Germline and somatic mutations in an oncogene: RET mutations in inherited medullary thyroid carcinoma. Cancer Res. 1996;56(6):1241-1243.

27. Zhou Y, Zhao Y, Cui B, et al. RET proto-oncogene mutations are restricted to codons 634 and 918 in mainland Chinese families with MEN2A and MEN2B. Clin Endocrinol (Oxf). 2007;67(4):570-576. doi:10.1111/j.1365-2265.2007.02927.x

28. Figlioli G, Landi S, Romei C, Elisei R, Gemignani F. Medullary thyroid carcinoma (MTC) and RET protooncogene: mutation spectrum in the familial cases and a meta-analysis of studies on the sporadic form. Mutat Res. 
2013;752(1):36-44. doi:10.1016/j.mrrev.2012.09.002

29. Dvoráková S, Václavíková E, Sýkorová V, et al. New multiple somatic mutations in the RET proto-oncogene associated with a sporadic medullary thyroid carcinoma. Thyroid. 2006;16(3):311-316. doi:10.1089/thy.2006.16.311
30. Moura MM, Cavaco BM, Pinto AE, et al. Correlation of RET somatic mutations with clinicopathological features in sporadic medullary thyroid carcinomas. Br J Cancer. 2009;100(11):1777-1783. doi:10.1038/sj.bjc.6605056 